Journal for ImmunoTherapy of Cancer

\section{Nanoemulsion adjuvantation strategy of tumor-associated antigen therapy rephrases mucosal and immunotherapeutic signatures following intranasal vaccination}

To cite: Huang $\mathrm{C}-\mathrm{H}$, Huang $\mathrm{C}-\mathrm{Y}$, Ho H-M, et al. Nanoemulsion adjuvantation strategy of tumor-associated antigen therapy rephrases mucosal and immunotherapeutic signatures following intranasal vaccination. Journal for ImmunoTherapy of Cancer 2020;8:e001022. doi:10.1136/jitc-2020-001022

- Additional material is published online only. To view please visit the journal online (http://dx.doi.org/10.1136/jitc2020-001022).

Accepted 28 August 2020

\section{Check for updates}

(c) Author(s) (or their employer(s)) 2020. Re-use permitted under CC BY-NC. No commercial re-use. See rights and permissions. Published by BMJ.

${ }^{1}$ Department of Food Science, National Taiwan Ocean University, Keelung, Taiwan ${ }^{2}$ National Institute of Infectious Diseases and Vaccinology, National Health Research Institutes, Miaoli, Taiwan ${ }^{3}$ Institute of Biotechnology, National Tsing Hua University, Hsinchu, Taiwan

${ }^{4}$ Graduate Institute of Biomedical Sciences, China Medical University, Taichung, Taiwan ${ }^{5}$ Graduate Institute of Medicine, Kaohsiung Medical University, Kaohsiung, Taiwan

Correspondence to Dr Ming-Hsi Huang; huangminghsi@nhri.org.tw

\section{ABSTRACT}

Background Emulsion adjuvants are a potent tool for effective vaccination; however, the size matters on mucosal signatures and the mechanism of action following intranasal vaccination remains unclear. Here, we launch a mechanistic study to address how mucosal membrane interacts with nanoemulsion of a well-defined size at cellular level and to elucidate the impact of size on tumorassociated antigen therapy.

Methods The squalene-based emulsified particles at the submicron/nanoscale could be elaborated by homogenization/extrusion. The mucosal signatures following intranasal delivery in mice were evaluated by combining whole-mouse genome microarray and immunohistochemical analysis. The immunological signatures were tested by assessing their ability to influence the transportation of a model antigen ovalbumin (OVA) across nasal mucosal membranes and drive cellular immunity in vivo. Finally, the cancer immunotherapeutic efficacy is monitored by assessing tumor-associated antigen models consisting of OVA protein and tumor cells expressing OVA epitope.

Results Uniform structures with $\sim 200 \mathrm{~nm}$ in size induce the emergence of membranous epithelial cells and natural killer cells in nasal mucosal tissues, facilitate the delivery of protein antigen across the nasal mucosal membrane and drive broad-spectrum antigen-specific T-cell immunity in nasal mucosal tissues as well as in the spleen. Further, intranasal vaccination of the nanoemulsion could assist the antigen to generate potent antigen-specific CD8+ cytotoxic T-lymphocyte response. When combined with immunotherapeutic models, such an effective antigenspecific cytotoxic activity allowed the tumor-bearing mice to reach up to $50 \%$ survival 40 days after tumor inoculation; moreover, the optimal formulation significantly attenuated lung metastasis.

Conclusions In the absence of any immunostimulator, only $0.1 \%$ content of squalene-based nanoemulsion could rephrase the mucosal signatures following intranasal vaccination and induce broad-spectrum antigen-specific cellular immunity, thereby improving the efficacy of tumorassociated antigen therapy against in situ and metastatic tumors. These results provide critical mechanistic insights into the adjuvant activity of nanoemulsion and give directions for the design and optimization of mucosal delivery for vaccine and immunotherapy.

\section{BACKGROUND}

Nasal spray or intranasal vaccination is considered a promising strategy for inducing mucosal protection against respiratory tract infections and systemic immunity with longlasting memories ${ }^{1}$; however, antigen applied to mucosal membranes generally induces minuscule immune responses owing to mucosal membrane barriers and immune tolerance. ${ }^{2}$ Another obstacle for intranasal vaccination derives from evidence pointing to the toxin-based nasal adjuvants posing a risk of inoculum invasion into the central nervous system. ${ }^{2}$ Therefore, the aid of a safe mucosal adjuvant with high efficacy is necessary for antigen recognition by the mucosal immune system and generation of broad-spectrum immune responses.

Emulsion adjuvants are a potent tool for effective vaccination against infectious disease and cancer. ${ }^{3-8}$ Despite progress in this field, no intranasal vaccine or immunotherapy formulated with this type of adjuvants is currently registered for human use to protect against or treat disease. Potential safety concerns and low immunity generated in a manner suitable for patients remain major challenges. Over the years, it has become clear that size is critical for adjuvant activities, and smallsized particles are considered more bioactive than large ones. ${ }^{9}$ One of the major explanations for this phenomenon is that particles with a smaller size have a larger surface area, which is beneficial for antigen loading and particle-cell interactions. ${ }^{10}$ Another explanation is that particles at the nanoscale are most effective for antigen delivery across 
mucosal surface barriers compared with larger sized ones. ${ }^{1011}$ With respect to immunological effects, different size ranges of particles may result in different levels and qualities of immune responses. Although the relationship between particle size and the generated immune profile remains controversial, some studies have substantiated that particles with a size ranging from 20 to $200 \mathrm{~nm}$ are mainly internalized by immune cells via endocytosis, leading to the dominant cellular immune responses. ${ }^{11}$ In contrast, particles in the size range of $0.5-5 \mu \mathrm{m}$ are usually targeted to phagocytic cells in vivo, resulting in the major humoral immune responses. ${ }^{11}$ Accordingly, the development of a particle-based mucosal adjuvant at the nanoscale is suggested as a potential strategy to deliver antigens across mucosal barriers and elicit concomitant cell-mediated immune responses.

We have previously optimized a ready-to-use emulsion adjuvant dubbed PELC, which is cored by squalene (an isoprenoid hydrocarbon with six unsaturated double bonds) and emulsified by Span85 (sorbitan trioleate) and a bioresorbable polymer poly(ethylene glycol)-

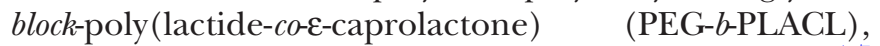
suspending in phosphate-buffered saline (PBS). ${ }^{4-7}$ Following parenteral injection, our results suggest that unsaturated squalene oil was more powerful than saturated squalane oil to induce reactive oxidative speciesmediated antigen uptake ${ }^{8}$; moreover, adjuvantation with the aid of PELC may be a prospective strategy to manipulate antigen-specific immune responses and to build on rational vaccine design and fabrication in prophylactic and therapeutic applications. ${ }^{5-7}$ However, intranasal

A

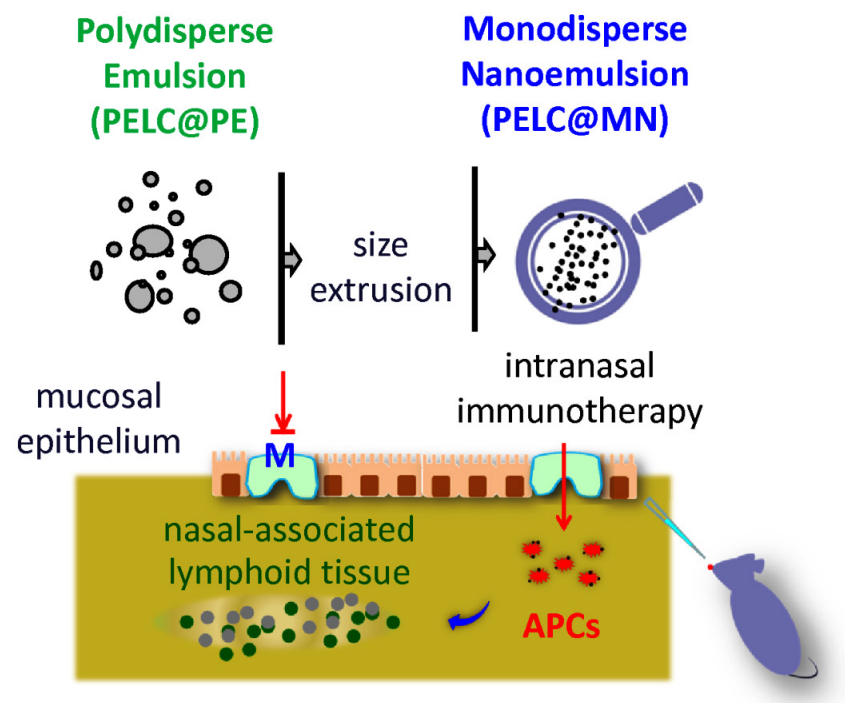

vaccination with PELC in the absence of immunomodulatory agents does not elicit forceful immune responses, ${ }^{45}$ and thus an appreciative condition for mucosal delivery should still be optimized.

Here we launch a mechanistic study that monodisperse nanoemulsion facilitates antigen transportation across the nasal mucosal membrane and thereby enhances vaccine efficacy, compared with free antigen vaccination (figure 1A). This investigation is brought to fruition by tailoring the PELC particles, originally polydisperse submicron structure, to monodisperse nanosized distribution by progressively passing through extruder membranes. We choice chicken ovalbumin (OVA) as model antigen to further understand the signatures of the monodisperse nanoemulsion interacting with mucosal membranes in mice and to elucidate their roles in vaccine immunogenicity following intranasal vaccination. In the beginning, we investigate whether monodisperse nanoemulsion can facilitate the transportation of antigen across the mucosal membrane to the site of immune induction, the nasal-associated lymphoid tissue (NALT),${ }^{12}$ compared with polydisperse ones. The immunological signatures of these formulations are then tested by assessing their ability to drive broad-spectrum mucosal and splenic cellular immunity via intranasal vaccination. Finally, the adjuvant efficacy is examined by assessing codelivery of OVA protein with monodisperse nanoemulsion to achieve a successful immunotherapy by delivering a stronger cytotoxic T lymphocyte (CTL) response to kill antigen-transfected cells, and the respective mode of adjuvant action is proposed.

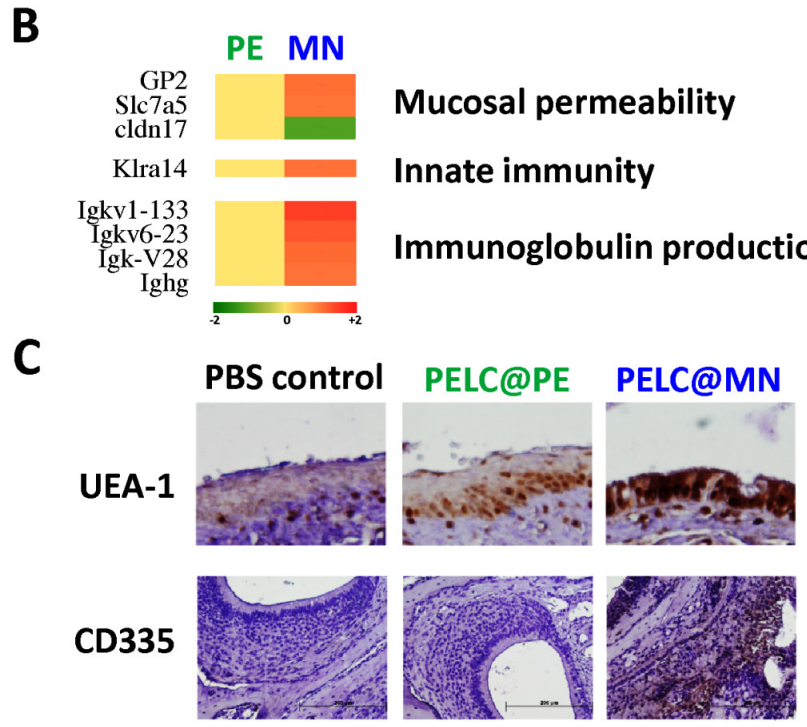

Figure 1 Tailoring emulsified particles to monodisperse nanosized distribution rephrases mucosal signatures following intranasal vaccination. (A) Schematic illustration of the monodisperse nanoemulsion enables the antigens to pass through the mucosal epithelium and facilitate the transportation of antigens into lymphoid tissues. (B) Microarray analysis of transcription profiles induced by emulsified particles 20 hours after administration. Genes with a fold change $\geq 1.5$ and $p<0.05$ compared with the PBS control. (C) Membranous (M) cell emergence and natural killer (NK) cell trafficking in nasal mucosa. Nasal mucosal tissues were harvested and phenotyped by immunohistochemical (IHC) staining. The brown signal around the blue nucleus indicates UEA-1+ and CD335+ cells, respectively (magnification, 400x). APCs, antigen-presenting cells. PBS, phosphatebuffered saline. 
MATERIALS AND METHODS

\section{Study design}

We propose/demonstrate that tailoring emulsified particles to the monodisperse nanosized distribution can play a role in facilitating the transportation of antigen across mucosal membrane to the NALT and thereby enhances vaccine efficacy, compared with the original polydisperse ones. All experiments were conducted independently at least twice. The production of antigen-specific immunity and tumor challenge studies were designed to evaluate the size matter of the emulsion adjuvants on the immunogenicity as well as tumor-associated antigen therapy in a mouse model with OVA as antigen. Pooled data are presented for the draining lymph node (LN) cells and T-cell immunity experiments to obtain sufficient replicates for each condition. The number of animals in each group was based on the minimum number of mice for statistical analysis and replicate experiments. Prior to treatment, the animals were randomized to minimize variances between groups. All materials injected into animals were determined to be free of mycoplasma.

\section{Chemicals, reagents, antibodies and cell lines}

Reagents and media for cell culture were purchased from Thermo Fisher Scientific (NY, USA) and GE Healthcare (Utah, USA). Reagents and standards for ELISA were purchased from R\&D Systems (MN, USA). Antibodies used for flow cytometric analysis were purchased from BioLegend (CA, USA). The EG7 cell line (American Type Culture Collection, CRL-2113) was maintained in complete RPMI 1640 medium supplemented with an aminoglycoside antibiotic Calbiochem G418 (0.4 mg/ mL, Merck, Darmstadt, Germany). The B16-F10-OVA cell line was maintained in complete Dulbecco's modified Eagle's medium supplemented with $0.4 \mathrm{mg} / \mathrm{mL}$ of G418.

\section{Emulsion preparation}

For PELC@PE preparation, a mixture comprising $120 \mathrm{mg}$ of diblock copolymer PEG-b-PLACL, $0.8 \mathrm{~mL}$ of PBS, 0.935 $\mathrm{mL}$ of squalene and $0.165 \mathrm{~mL}$ of Span 85 was first emulsified by means of a homogenizer (Polytron PT 2500E, Kinematica, Swiss), as previously described..$^{5-7}$ Then, 200 $\mu \mathrm{L}$ of the stock emulsion was redispersed into $1800 \mu \mathrm{L}$ of PBS in a test tube, and allowed to mix the emulsified suspension using a rotator at a mild condition ( $5 \mathrm{rpm}$ ) for 1 hour. Next, $10 \mu \mathrm{L}$ aliquot of a specimen was added to $990 \mu \mathrm{L}$ of PBS, rendering a final diluted sample (referred to as PELC@PE).For PELC@MN preparation, PELC@PE suspension was passed through polycarbonate membranes (Whatman Nuclepore Track-Etched Membranes, GE Healthcare, Darmstadt, Germany) mounted in a miniextruder fitted with two $1.0 \mathrm{~mL}$ gas-tight syringes. Typically, 11 passages were performed to achieve a monodisperse distribution and to avoid contamination with the sample (an odd number of passages) that might not have passed through the membrane. After progressively passing through 1.0, 0.4, 0.2, and $0.1 \mu \mathrm{m}$ membranes, the emulsified particles were obtained with monodisperse distribution and at the nanoscale (referred to as PELC@ $\mathrm{MN})$. The particle size distribution was measured by a particle size analyzer (Nano ZS, Malvern, Brookhaven Instruments, NY, USA). The microscopic aspects were monitored using an optical microscope (Olympus DP70) and a transmission electron microscopy (H-7650, Hitachi, Japan).

\section{Mice}

$\mathrm{BALB} / \mathrm{c}$ and C57BL/ 6 female mice at age of 5 weeks were obtained from the National Laboratory Animal Breeding and Research Centre (Taipei, Taiwan) and acclimatized for 1 week at the Laboratory Animal Centre of the National Health Research Institutes (Miaoli County, Taiwan) prior to use.

\section{Nasal mucosal gene expression analysis}

$\mathrm{BALB} / \mathrm{c}$ mice ( $\mathrm{n}=3$ per group) were intranasally administered in both nostrils with $10 \mu \mathrm{L}$ per nostril of PBS, PELC@PE or PELC@MN. Nasal mucosa was harvested from mice at 20 hours after administration. Parts of the mucosal samples were used for total RNA extraction with TRI Reagent (Sigma-Aldrich) according to the manufacturer's instructions, and the other parts were prepared for immunohistochemical (IHC) staining. Prior to hybridization, the quality and integrity of the RNA samples were determined using an Agilent 2100 Bioanalyzer. Hybridization was performed using Mouse Gene 2.0 ST array (Affymetrix, CA, USA), and the microarrays were scanned by a Molecular Devices GenePix 4000B Scanner according to the manufacturer's guidelines. Gene expression values were analyzed by gene set enrichment analysis.

\section{IHC staining of nasal mucosal tissues}

IHC was performed on 4-5 $\mu \mathrm{m}$ sections of each paraffin block. The sections were deparaffinized and rehydrated by sequentially immersing into xylene and ethanol as previously described. ${ }^{6}$ For antigen retrieval, the hydrated slides of nasal mucosal and tumor tissues were immersed in Trilogy at $90^{\circ} \mathrm{C}$ for $30 \mathrm{~min}$, followed with $3 \%$ hydrogen peroxide $\left(\mathrm{H}_{2} \mathrm{O}_{2}\right)$ for 15 min to block the endogenous peroxidase activity. After washing with PBS, the slides were further blocked with $2.5 \%$ normal horse serum for 1 hour. The primary antibodies, anti-CD3, anti-CD4, anti-CD8, anti-CD335 NKp46 (BioLegend) and anti-UEA-1 (MBL, IL, USA), were applied, and the sections were then incubated at room temperature for 1 hour. After washing with PBS, the sections were further incubated with the peroxidase polymer detection kit (Nichirei Bioscience, Tokyo, Japan) for 1 hour. Finally, the slides were visualized by DAB staining, followed by hematoxylin counterstaining. The IHC-positive signals were observed and analyzed at a 400-fold magnification using an Olympus DP70 microscope. The number of IHC-positive signals and area of positive signal were quantified using the ImageJ image processing and analysis program (Bethesda, MD, USA). Three measurements per tissue section and three to six 
sections per group were analyzed at 200-fold and 400-fold magnification.

\section{Detection of OVA transportation}

$\mathrm{BALB} / \mathrm{c}$ mice ( $\mathrm{n}=3$ per group) were intranasally administered with OVA alone, OVA plus PELC@PE or OVA plus PELC@MN. The mice were sacrificed at 0,4 and 20 hours after administration. The harvested nasal mucosal tissues were washed with saline and then fixed for 24 hours with $10 \%$ neutral buffered formalin. Tissue blocks and slides were prepared as mentioned above, and the anti-OVA antibody (BioLegend) was used for staining of OVA in mucosal tissues.

\section{Vaccination and immunoassays}

For immunological evaluation, BALB/c mice were vaccinated via the intranasal route with OVA formulations $(100 \mu \mathrm{g} /$ dose, $20 \mu \mathrm{L})$ once a week for 3 weeks. One week after the final vaccination, the mice ( $n=3$ per group) were sacrificed to collect nasal mucosal tissues and spleen samples for further experiments.

Blocks and slides of nasal mucosal tissue were prepared as mentioned above, and anti-CD3, anti-CD4 and antiCD8 antibodies (BD Biosciences, CA, USA) were used for IHC staining of T cells in the mucosal tissues.

Splenocyte suspensions were collected from the mouse spleen as described previously. ${ }^{6}$ Splenocyte suspensions $\left(5 \times 10^{6}\right.$ cells $\left./ \mathrm{mL}\right)$ were cultured in the presence of OVA $(10 \mu \mathrm{g} / \mathrm{mL})$. At 24 hours, cell pellets in each group were prepared for extraction of total RNA using TRI Reagent (Sigma) according to the supplier's instruction. The steady-state mRNA expression levels, T-bet and ROR $\gamma t$, were measured by reverse transcription PCR in a manner similar to our previous study. ${ }^{6}$ The results are shown as the density ratio of the interest gene to the reference standard ( $\beta$-actin). At 72 hours, the supernatants were collected from triplicate cultures and tested for interferon gamma (IFN- $\gamma$ ) and interleukin 17 (IL-17) concentrations (DueSet ELISA Development Kit, R\&D Systems) following the manufacturer's instructions.

\section{Phenotype and functional activation of CTLS}

The antigen-specific CTL response was determined by analyzing the frequency of SIINFEKL-MHC-I tetramer+ CD8+ T cells. C57BL/ 6 mice ( $n=4$ per group) were intranasally vaccinated with different OVA formulations (100 $\mu \mathrm{g}$ per dose, $20 \mu \mathrm{L}$ ) once a week for 3 weeks. One week after the final vaccination, splenocytes $\left(5 \times 10^{6}\right.$ cells $\left./ \mathrm{mL}\right)$ harvested from vaccinated mice were treated with SIINFEKL $(1 \mu \mathrm{g} / \mathrm{mL})$ for 24 hours and stained with $\mathrm{H}-2 \mathrm{~Kb}-$ restricted SIINFEKL tetramer, fluorescein isothiocyanate (FITC)-conjugated anti-CD8 and phycoerythrin (PE)conjugated anti-CD107a antibodies on ice bath for 30 min. Samples were then examined by flow cytometry (LSRII; BD Immunocytometry Systems, CA, USA), and CD8+ T cells were gated for dot plot analysis of CD107a (PE) versus tetramer (APC, allophycocyanin). The mean fluorescence intensity (MFI) of CD107a in gated CD8+H-2Kb/SIINFEKL+ cells was also determined.

\section{Tumor challenge study}

For in situ tumor model, a total of $2 \times 10^{5}$ EG7 tumor cells per mouse were first inoculated subcutaneously into the flank of C57BL/ 6 mice ( $n=6$ per group). On the appearance of palpable tumors, the mice were intranasally vaccinated once a week for 3 weeks with $100 \mu \mathrm{g}$ of OVA, non-formulated or formulated with candidate adjuvants. Tumor sizes were measured in two vertical dimensions using a digimatic caliper twice per week. Tumor volumes were calculated following the formula: (length $\times$ width $\times$ width)/2. For ethical issues, the mice were euthanized when they experienced severe faintness or when the tumor volume exceeded $2000 \mathrm{~mm}^{3}$.

For the lung melanoma metastatic model, C57BL/ 6 mice ( $n=6$ per group) were injected intravascularly with B16-F10-OVA cells $\left(5 \times 10^{5}\right.$ cells/mouse $)$. One week later, the mice were intranasally vaccinated once a week for 3 weeks with $100 \mu \mathrm{g}$ of OVA, non-adjuvanted or adjuvanted with designed emulsions. One week after the final vaccination, the mice were sacrificed, and the lung was isolated for pathological observation.

\section{Statistics}

The statistical difference between each treatment group was assessed by Dunnett's two-tailed t-test using GraphPad Prism V.5.02 (GraphPad Software). $\mathrm{P}<0.05$ was defined as significant. The median survival in tumor challenge study was calculated using the Gehan-Breslow-Wilcoxon method.

\section{RESULTS \\ Tailoring emulsified particles to monodisperse nanosized distribution rephrases mucosal signatures following intranasal delivery}

PELC suspensions issued from homogenization initially formed emulsified particles at the submicron scale with polydisperse distribution (termed PELC@PE for the remainder of this article). Such liquid-liquid colloids were soft and deformable so that the size could be controlled by passage through an extruder membrane (online supplemental figure S1). After sequential extrusion through membranes, as expected, particles with monodisperse nanosized distribution (termed PELC@ $\mathrm{MN}$ for the remainder of this article) were observed with an average diameter of about $200 \mathrm{~nm}$, which is consistent with the scale of nanoemulsion. ${ }^{13}$ In vitro results from our preliminary investigations show that monodisperse nanoemulsion with a well-defined size can dramatically induce activation of murine bone marrow-derived dendritic cells, compared with polydisperse emulsion (online supplemental figure S2). Subsequently, we plan to explore the adjuvant potency of the prepared emulsions to evaluate their remarkable mucosal activity and immunotherapeutic efficacy. 
First, the innate immunological signatures following intranasal delivery were evaluated based on the impact of the monodisperse nanoemulsion on gene expression changes in nasal mucosal tissues. To achieve this goal, mice intranasally received $20 \mu \mathrm{L}$ of PELC@PE or PELC@MN diluted in PBS, and large-scale gene expression profiling of local mucosal tissues was performed at 20 hours after administration by whole-mouse genome microarray analysis. Among total genes screened, both PELC@PE and PELC@MN emulsions regulated a common set of 66 genes with a fold change $\geq 1.5$ and $\mathrm{p}<0.05$ compared with the PBS treatment group (online supplemental tables S1-S3). Interestingly, PELC@MN regulated a larger number of genes (230) compared with PELC@PE (197), which were associated with categories including cell/mucosal permeability, immune responses, fatty acid metabolism, neurotransduction, oxidation and reduction, xenobiotic excretion, and so on (online supplemental table S4). Several genes associated with mucosal permeability, glycoprotein 2 (GP2), ${ }^{14}$ Slc7a $5,{ }^{15}$ cldn $17,{ }^{16}$ innate immunity (Klra14) ${ }^{17}$ and immunoglobulin production (Igkv1-133, Igkv6-23, Igk-V28, Ighg) ${ }^{18}$ were significantly regulated by PELC@MN compared with PELC@PE (figure 1B).

Concerning the results of microarray analysis, PELC@ $\mathrm{MN}$ significantly upregulated the expression of numerous genes associated with mucosal permeability. Among these genes, GP2 was exclusively expressed on membranous (M) cells, which are specialized mucosal epithelial cells found in the mucosa-associated lymphoid tissues of nasal and intestinal mucosa and able to transport antigens across epithelial membrane for further interaction with immune cells. ${ }^{14}$ To further study the impact of size on inducing the emergence of $\mathrm{M}$ cells, the harvested nasal tissues were also assessed for IHC staining, and the slides were stained with anti-UEA-1, a common biomarker for M cells. ${ }^{14}$ As shown in figure 1C, more UEA-1+ cells were detected in PELC@MN-treated mice than in PELC@ PE-treated and PBS-treated mice.

On the other hand, PELC@MN also altered the gene expression of Klra14, which represents a lectin-like receptor subfamily A expressed on natural killer (NK) cells. ${ }^{17}$ Therefore, we further investigated the impact of PELC@MN on the recruitment of NK cells to the nasal mucosa by IHC staining of CD335, a cytotoxicityactivating receptor that may be highly specific to NK cells. ${ }^{19}$ As shown in figure 1C, more CD335+ cells were detected in PELC@MN-treated mice than in PELC@ PE-treated and PBS-treated mice. The findings from microarray analysis and IHC staining suggest that the increased mucosal permeability induced by PELC@MN may be associated with the greater number of $\mathrm{M}$ cells. In addition, PELC@MN may be used as a powerful tool in mucosal delivery of vaccine antigens and in the activation of innate immunity by increasing the number of activated NK cells.
Monodisperse nanoemulsion facilitates delivery of protein antigen across nasal mucosal membrane and drives broadspectrum antigen-specific immunity

We next planned to assess the augmentation of mucosal permeability by PELC@MN in relation to adaptive immunity. We attempted to address whether PELC@MN could facilitate protein antigen transportation across the nasal mucosal membrane into NALT. Mice received intranasally with $50 \mu \mathrm{g}$ of OVA in both nostrils with either antigen alone or formulated with PELC@PE or PELC@ MN. The nasal mucosal tissues were harvested at 0,4 and 20 hours after administration and IHC stained with OVA antibodies. No OVA-positive signal was recognized in the IHC images of OVA-treated mice and PELC@PE-formulated OVA-treated mice within 20 hours (figure 2A), indicating an absence of OVA transportation across the nasal membrane. Brown signals (ie, OVA positive) were clearly induced in the nasal mucosal tissues of mice treated with PELC@MN-formulated OVA at 4 hours and increased progressively at 20 hours, that is, OVA transportation was strongly influenced by the presence of the PELC@ MN particles, suggesting that PELC@MN could protect against the removal of protein antigen from the nasal cavity for at least 20 hours and deliver antigen across the mucosal barrier and into NALTs.

Cellular immunity has the prospect to play an important role in mucosal protection; there is evidence that memory T-cell populations, which are inherent in mucosal tissues, can respond rapidly and directly to infiltrating pathogens. ${ }^{19} 20$ Thus, we investigated the impact of the monodisperse nanoemulsion on populations of mucosal $\mathrm{T}$ cells following intranasal vaccination. As shown in figure 2B and online supplemental figure S3, OVA alone had no impact on the number of CD3+, CD4+ and CD $8+\mathrm{T}$ cells in the nasal mucosal tissues compared with the PBS control group. Notably, vaccination with PELC@MN-adjuvanted OVA induced a dramatic increase in the numbers of T-cell subsets in the surface as well as the lamina propria of nasal mucosal tissues; however, the effect was rather diminished when the adjuvant was replaced with PELC@ PE. We further confirmed the importance of the monodisperse nanoemulsion on the functional activation of splenic $\mathrm{T}$ cells, which were harvested from the vaccinated mice following incubation of cells in vitro with OVA antigen (figure 2C). Concerning the cytokine secretion, PELC@MN was the most potent inducer of IFN- $\gamma$, a predominant $\mathrm{T}$ helper type 1 (Th1) cytokine, whereas IFN- $\gamma$ secretion induced by PELC@PE-adjuvanted OVA was measured at the same level as OVA alone. Similarly, PELC@MN-adjuvanted OVA enhanced notable IL-17 secretion (the major Th17 cytokine) compared with those in the non-adjuvanted and PELC@PE-adjuvanted groups. This finding indicated that OVA at the dose of $100 \mu \mathrm{g}$ did not elicit adaptive responses; in addition, the presence of PELC@PE did not alter the situation. Concordantly, the mRNA expression levels of T-bet (Th1 type) and ROR $\gamma \mathrm{t}$ (Th17 type) were also significantly elevated in the PELC@ MN-adjuvanted OVA group. Based on the above results, 
A

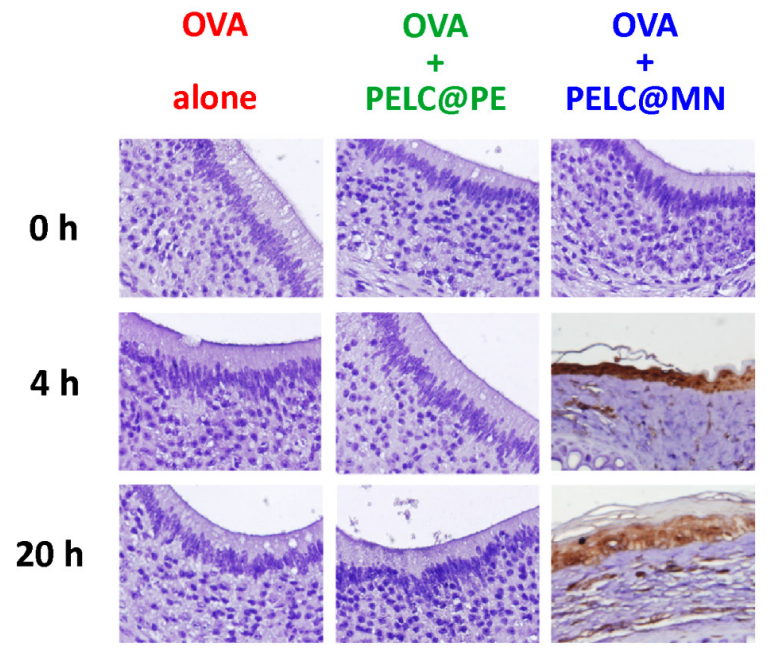

B

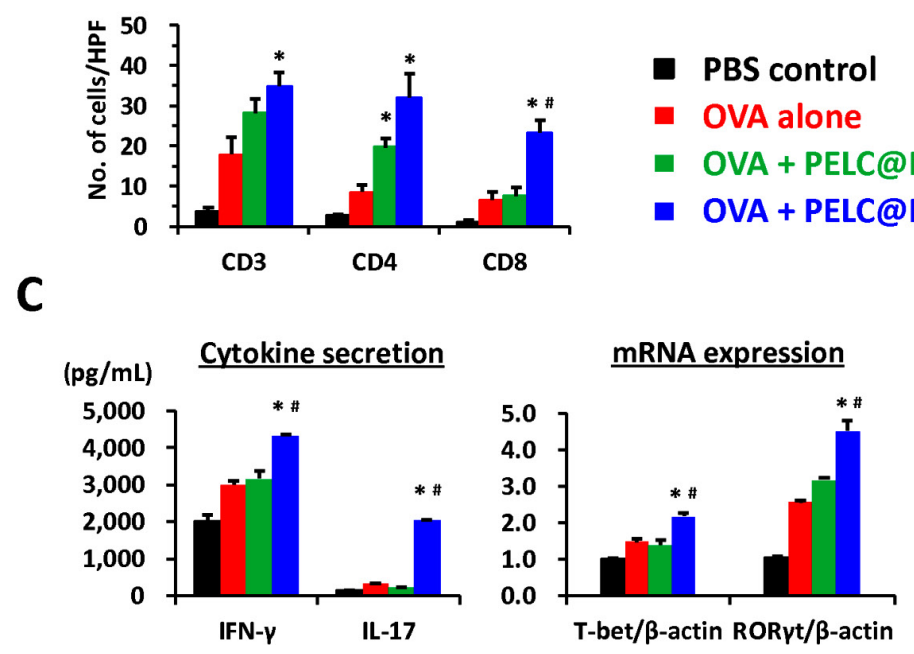

Figure 2 Monodisperse nanoemulsion facilitates the delivery of protein antigen across nasal mucosal membranes and drives broad-spectrum antigen-specific immunity in mice. (A) Transportation of OVA across nasal mucosal membranes. The nasal mucosal tissues were harvested from the treated mice and immunohistochemical (IHC) stained with OVA antibodies. The brown signals indicate OVA-positive signals (magnification, 400x). One week after the final administration, the mice ( $\mathrm{n}=3$ ) were sacrificed, and then nasal mucosal tissues and spleen were harvested for T-cell immunoassays. (B) T-cell subsets in nasal mucosal tissues. Observe microscopic fields using a power of $400 \times$ (see also online supplemental figure S3). (C) T-cell immunity in the spleen. The cell pellets were collected for mRNA expression of T-bet, ROR $\gamma$ t and $\beta$-actin by reverse transcription PCR (RT-PCR), respectively. The supernatants were collected to measure IL-17 and IFN- $\gamma$ secretion by ELISA. Data are expressed as the means \pm SEM. The results are representative of two to three independent experiments. ${ }^{*}<<0.05$ compared with 0 VA alone. $\# \mathrm{P}<0.05$ compared with the polydisperse emulsion group. HPF, high-power field; IFN- $\gamma$, interferon gamma; IL-17, interleukin 17; OVA, ovalbumin; PBS, phosphate-buffered saline.

emulsified particles with monodisperse distribution and at the nanoscale should be regarded as simple delivery systems for the delivery of antigen across the mucosal barrier and into NALTs and can play an active role in mediating Th1 and Th17 immune responses.

\section{Monodisperse nanoemulsion assists antigen in generating potent antigen-specific CD8+ CTLs as well as antitumor} ability when combined with tumor-associated antigen therapy The phenotype and functional activation of CD8+ CTLs are critical characteristics in the clearance of virusinfected cells and in the defense against cancers. ${ }^{21}$ To test the potency of the monodisperse nanoemulsion to trigger the antigen-specific CTL response, we analyzed the frequency of SIINFEKL-MHC-I tetramer+ CD8+ $\mathrm{T}$ cells. OVA-restimulated splenocytes were stained with a fluorescence-labeled anti-CD8 antibody and a fluorescence-conjugated SIINFEKL/MHC-I tetramer (where SIINFEKL is the H-2Kb-restricted OVA MHC class I epitope).$^{21}$ As the benchmark, we also evaluated the elicitation of antigen-specific CTLs by analyzing the expression of CD107a on CD8+H-2Kb/SIINFEKL+ cells. In the literature regarding $\mathrm{CD} 8+\mathrm{T}$-cell degranulation, CD107a expression is closely associated with both cytokine secretion and cell-mediated lysis of target cells. ${ }^{22}$ As shown by flow cytometric analysis (figure 3A), the percentage of $\mathrm{CD} 8+\mathrm{H}-2 \mathrm{~Kb} / \mathrm{SIINFEKL}+$ cells in splenocytes was approximately $1 \%$ in mice vaccinated with OVA alone and PELC@PE-adjuvanted OVA. Notably, the population of $\mathrm{CD} 8+\mathrm{H}-2 \mathrm{~Kb} / \mathrm{SIINFEKL}+$ cells was significantly increased to $5 \%$ in the vaccinated PELC@MN-adjuvanted OVA group, indicating that PELC@MN is able to induce the expansion of antigen-specific CD8+ CTLs via intranasal delivery. In addition, the MFI of the activation marker CD107a on gated CD8+H-2Kb/SIINFEKL+ cells was markedly increased from 70 to 551 in mice vaccinated with PELC@MN-adjuvanted OVA (figure 3B). However, the potency was slightly reduced when the adjuvant was replaced with PELC@PE. These results substantiated that PELC@MN emulsion as a mucosal adjuvant is capable of driving the expansion and activation of antigen-specific CTLs.

We conducted two murine models to evaluate whether the antigen-specific cytotoxicity in vivo reflected effective clearance of antigen-transfected cells. First, we applied an in situ tumor consisting of OVA protein/EG7 cells (OVA-transfected EL4 murine thymoma cells) as a tumor antigen/tumor cell model. ${ }^{6}$ All mice were first inoculated subcutaneously with EG7 tumor cells, and then they were vaccinated intranasally with OVA antigen, alone or adjuvanted with either PELC@PE or PELC@MN on days 7, 14 and 21. Figure 3C,D shows the monitored tumor volume and survival rate. The tumors grew progressively in the PBS control group (to mimic the tumor-bearing mice without receiving any treatment), and the mice started to die within 30 days. All mice that received non-adjuvanted OVA died before day 42, indicating that no protection for the mice received OVA alone. Vaccination of mice with OVA plus PELC@PE provided a better protective 
A

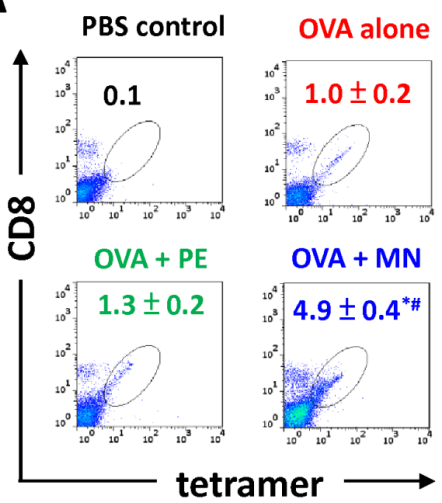

B

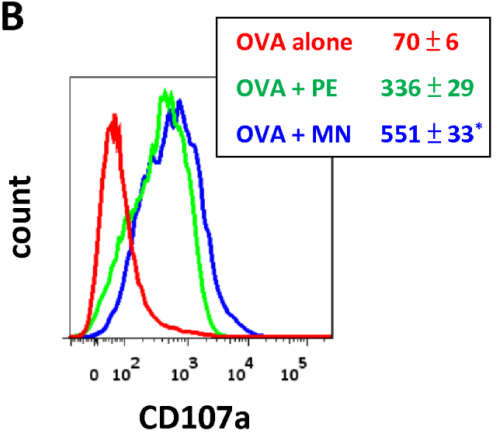

C

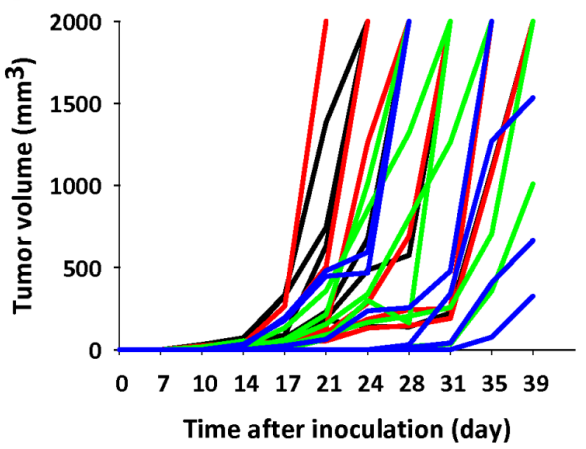

D

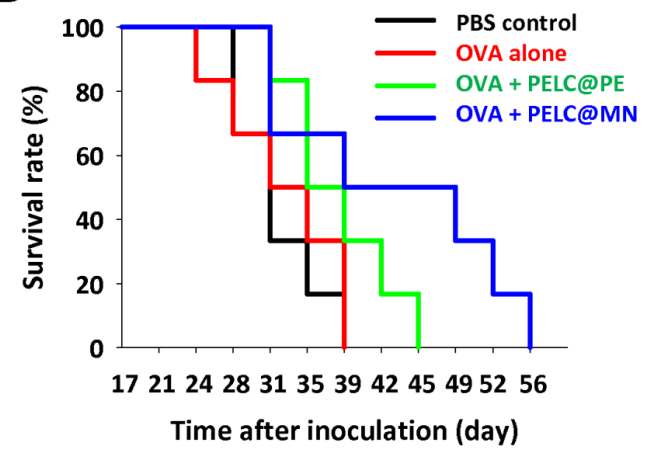

E

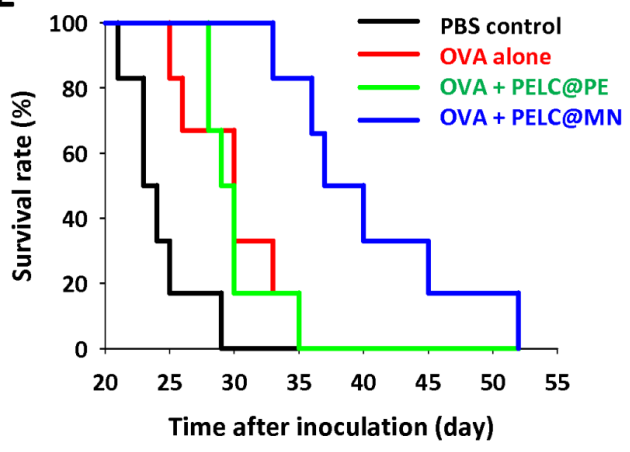

$\mathbf{F}$

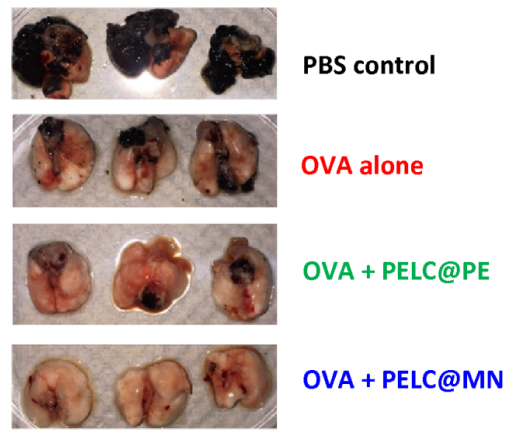

Figure 3 Intranasal delivery of monodisperse nanoemulsion could generate potent antigen-specific CD8+ cytotoxic T lymphocytes (CTLs) as well as clearance of antigen-transfected cells when combined with tumor-associated antigen therapy. Expansion and activation of antigen-specific CD8+ T cells. (A) The antigen-specific CTL response was determined by SIINFEKLMHC-I tetramer staining and flow cytometry. (B) The mean fluorescence intensity (MFI) of CD107a on CD8+ T cells. Data are expressed as the mean \pm SEM from individual murine splenic sample. Intranasal delivery of PELC@MN-adjuvanted OVA inhibits tumor in situ. The mice $(n=6)$ were first subcutaneously inoculated in the flank with $E G 7$ tumor cells $\left(1 \times 10^{5}\right.$ cells per mouse). Seven days after tumor cell inoculation, the mice in each group were intranasally vaccinated with $100 \mu \mathrm{g}$ per dose OVA protein without or with either PELC@PE or PELC@MN on days 7, 14 and 21. (C) Tumor volume and (D) survival rate. Intranasal delivery of PELC@MN-adjuvanted OVA inhibits lung metastasis. The mice $(n=6)$ were intravascularly inoculated with B16-F10-OVA cells $\left(5 \times 10^{5}\right.$ cells per mouse). One week later, the mice were intranasally vaccinated once a week for 3 weeks with OVA (100 $\mu g$ per dose), alone or adjuvanted with either PELC@PE or PELC@MN. (E) The survival rate was monitored two to three times per week. (F) Lung tissues at day 28 after inoculation of tumor cells. ${ }^{*} \mathrm{P}<0.05$ compared with OVA alone. $\# \mathrm{P}<0.05$ compared with the PELC@PE group. OVA, ovalbumin; PBS, phosphate-buffered saline.

effect than those treated with OVA alone; however, it still not eradicates the inoculated EG7 cells. It is worthy to note that PELC@MN was better able than PELC@PE to broaden the immunotherapeutic efficacy of tumorassociated antigen (OVA) and to prolong the median survival of tumor cell (EG7)-bearing mice from 37 to 44 days. Last but not least, only the treatment with PELC@ MN-adjuvanted OVA allowed the mice to reach up to $50 \%$ survival 40 days after tumor inoculation.

We next targeted immunotherapy for the treatment of cancer cell migration. In this approach, B16-F10-OVA (a stable transfectant murine OVA-expressing B16-F10 melanoma) was inhibited by vaccination with OVA in a metastatic cancer model. ${ }^{7} \mathrm{C} 57 \mathrm{BL} / 6$ mice that were intravascularly inoculated with B16-F10-OVA and then intranasally vaccinated three times at a 1-week interval with PELC@MN-adjuvanted OVA showed inhibited growth in tumor-bearing mice, compared with PELC@PE-adjuvanted OVA or OVA alone (figure 3E). Within 28 days, lethality started in the group of mice that did not get any treatment (PBS control). Although vaccination with OVA alone or PELC@PE-adjuvanted OVA alleviated lung metastasis, all the mice in these two groups died before day 35. Notably, a remarkably small tumor volume and impressively high survival rate were observed in the group of mice who were vaccinated with PELC@ MN-adjuvanted OVA (figure 3F), indicating that the aid of PELC@MN provided better protective capacity. These results demonstrate that intranasal vaccination of PELC@ MN-adjuvanted OVA induced OVA-specific CTLs, which potentially killed OVA epitope-expressing cancer cells, suggesting that PELC@MN is a promising adjuvant for the development of an antitumor immunotherapeutic vaccine.

\section{DISCUSSION}

Based on the mechanisms of action, mucosal adjuvants could be classified into three categories, including immunostimulators, particulate adjuvants, and their combinations. ${ }^{1-5}$ Immunostimulators, such as toll-like receptor agonists and bacterial toxins, have been assessed as 
mucosal adjuvants for a long time ${ }^{323}$; however, those made by the aforementioned compounds may sometimes be bombarded with medical risks for massive vaccination. ${ }^{3}$ Another concern is that mucosal epithelium is sometimes a barrier for targeting immune cells. ${ }^{3}$ To this, several delivery systems have been designed to transport antigens across mucosal barrier with diminutive obstruction and degradation by the mucociliary clearance system, mucin, and digestive enzyme. ${ }^{23}{ }^{24}$ In recent decades, the development of particulate adjuvants at nanoscale, such as nanoemulsions, liposomes, micelles, and metal particulates, has attracted large amounts of attention due to their properties of small size, large surface area, and improved solubility and interactions with the biological environment. $^{2526}$

The intake of fats or fatty acids may regulate or stimulate immune responses, such as lymphocyte proliferation, cytokine production, and phagocytosis. ${ }^{27}$ Such regulation of immune responses may result from several factors, but the change in the cell membrane due to the incorporation of lipid is considered the major point. ${ }^{28}$ Nevertheless, the intake of large oil globules may lead to non-specific immune reactions, such as delayed-type hypersensitivity, activation of NK cells and phagocytes, among others. ${ }^{29}$ Thus, some studies suggest that PEGylation may be a generic strategy for the lipid squalene, enabling the efficient formation of nanoparticles in buffer solution by self-assembly. ${ }^{30}$ Another strategy is to formulate the selected fats or fatty acids in the form of emulsions into vaccines to increase their immunogenicity and efficacy. Essentially, the good dispersion of oil drops in a vaccine formulation is crucial for the stability of the formulation, vaccine administration, evoking immune responses and attenuating local reactions. ${ }^{31}$ The oil-inwater emulsion adjuvant MF59 contains metabolizable squalene as the oily core, a lipophilic emulsifier Span85 and a hydrophilic Tween80 (polyoxyethylene sorbitan monooleate) suspended in buffer solution. MF59 has previously been demonstrated to be an effective adjuvant for parenteral injection but was not an effective adjuvant for intranasal vaccination with herpes simplex virus type 2 recombinant glycoprotein D2. ${ }^{23}$ Although the adjuvant mechanism of MF59 following intranasal vaccination is not clear, the authors suggested physically associating the antigen with the adjuvant is necessary. ${ }^{23}$ It has been reported that intranasal vaccination of vaccine candidates with a nanoemulsion containing cationic compound cetylpyridinium chloride induces strong and protective immune responses in rodents and ferrets towards the invaded pathogens. ${ }^{32} 33$ The potential mechanisms of action include sustained antigen adhesion to the mucosal membrane, induction of nasal epithelial cell apoptosis and heterogeneous cytokine production, transcellular antigen uptake in nasal epithelial cells and dendritic cells activation/trafficking to NALT. ${ }^{32-34}$ In the present study, we also substantiated that monodisperse squalene-cored emulsion at the nanoscale, that is, PELC@MN, sustained antigen retention at mucosal tissues for at least 20 hours and transported antigen across the mucosal barrier (figure 2A).

In our previous studies, we demonstrated that the addition of immunostimulators, for example, recombinant flagellin, ${ }^{4}$ and synthetic peptide analog LD-indolicidin, ${ }^{5}$ into the PELC emulsion is necessary for successful intranasal vaccination against influenza virus. Herein, intranasal vaccination with PELC@MN could elicit potent cell-mediated immunity, especially Th1 and Th17 immune responses, without using any immunostimulator or cationic surfactant. Most importantly, PELC@ MN could even increase the number of NK cells at the NALT, activate CD8+ CTLs, and attenuate lung metastasis, providing the complementary information of the potential of monodisperse nanoemulsions for cancer immunotherapy. Similar to cationic emulsions, PELC@ MN increased heterogeneous cytokine mRNA expression at mucosal tissues. The effects of PELC@MN on delivering antigen across mucosal membrane and eliciting immune responses are closely associated with the elevated permeability of the mucosal membrane. Based on the results of microarray analysis, the induction of epithelial cell death is considered a potential mechanism for increasing mucosal permeability and heterogeneous cytokine production. Merging the results of the microarray analysis and IHC staining, the elevated permeability may be due to the increase in transcellular and/or paracellular transport. In the transcellular pathway, the increase of nasal mucosal permeability is supported by the expression of M cells (figure 1B). On the other hand, the paracellular pathway involves the transport of protein antigen through the tight junctions between the epithelial cells, which is highly restricted for smaller particles (online supplemental figure S1). These findings were in agreement with literature data, which demonstrated that nanoparticles could surmount nasal epithelial barrier, so as that antigen presenting properties can be initiated. ${ }^{1125}$ Regarding this, further studies are warranted to verify the speculated mechanism of action of PELC@MN at the molecular level.

It has been demonstrated that the cytotoxic effects of NK cells and CD8+ CTLs contribute to protection against tumor metastasis. ${ }^{35}$ Previous studies in IL-17-deficient mice have reported that the augmented growth and metastasis of tumor potentially resulted from the diminished number of NK cells and tumor-specific IFN- $\gamma+$ $\mathrm{T}$ lymphocytes in tumor microenvironment as well as draining lymph nodes. ${ }^{36}$ Accordingly, the adjuvant activity of PELC@MN against lung metastasis may be mediated by its effects on elevating the number of NK cells and inducing the activation of Th1, Th17 and CTL cells. With this potential in mind, further investigations are underway to launch the translation of the nanoemulsion adjuvantation strategy to human clinical trials for elucidating the feasibility of nanoemulsion as mucosal adjuvants and/or spraying delivery for the prevention and treatment of infectious diseases and cancers. Research is needed to verify whether squalene nanoemulsion has 
similar effects in a human tumor xenograft model based on HLA transgenic mouse. With the aim of extending our results to enhance the efficacy of immunotherapy candidates in clinical applications, the research setting is rationally designed to investigate immunotherapeutic treatments comprising tumor-associated antigen therapy and immune checkpoint blockade therapy effectively reinforce each other via codelivery of tumor-associated antigens/immune checkpoint inhibitors with an optimal formulation towards enrichment of the immune microenvironments at local vaccination tissues, ipsilateral draining lymph nodes and tumor bed, thereby synergistically integrating the efficacy of individual cancer therapy. Such applications require nanoemulsion production at pilot scale in a consistent and reproducible manner which will be accomplished by homogenization with a high-shear microfluidized process.

\section{CONCLUSIONS}

In this study, we provided the comprehensive information demonstrating that tailoring emulsified particles to monodisperse nanosized distribution (assigned as PELC@MN) could rephrase mucosal and immunotherapeutic signatures following intranasal vaccination. Intranasal vaccination with tumor antigen adjuvanted with a squalene-cored PELC@MN significantly attenuated tumor growth and lung metastasis, probably due to the activation of innate immunity and cell-mediated immune responses. This hypothesis can be supported by several lines of evidence. First, intranasal vaccination with PELC@MN upregulated mucosal permeability, providing critical insights into the main action modes of PELC@ $\mathrm{MN}$ as mucosal adjuvant. Second, the numbers of M cells and NK cells at mucosal sites were increased in mice after intranasally vaccinated with PELC@MN-adjuvanted OVA. Third, intranasal vaccination with PELC@MN-adjuvanted OVA markedly induced the activation of CD8+ cytotoxic lymphocytes as well as Th1 and Th17 cells, and thereby improved the efficacy of cancer immunotherapy against in situ and metastatic tumors. These results provide critical mechanistic insights into the adjuvant activity of monodisperse nanoemulsion and give directions for the design and optimization of mucosal delivery for vaccine and immunotherapy.

\section{Twitter Ming-Hsi Huang @huangminghsi@}

Acknowledgements PTL carried out his master's thesis research under the auspices of the Graduate Program of Biotechnology in Medicine, National Tsing Hua University and National Health Research Institutes. The authors are grateful to MOST for awarding a postdoctoral research scholarship to $\mathrm{CHL}$ (108-2811-B-400-519).

Contributors $\mathrm{CHH}$ performed the experiments, analyzed the data and wrote the paper. CYH, HMH, CHL, and PTL performed the experiments. SCW and SJL conceived the experiments. MHH managed the whole project, conceived the experiments and wrote the paper. All authors contributed to manuscript preparation.

Funding This work was supported by grant 109A1-IVPP19-014 from the National Health Research Institutes of Taiwan and grant 106-2314-B-400-016-MY3 from the Ministry of Science and Technology (MOST) of Taiwan.

\section{Competing interests None declared.}

\section{Patient consent for publication Not required.}

Ethics approval All animal studies were conducted in accordance with the established institutional guidelines and the approval protocols from the IACUC review board of NHRI (NHRI-IACUC-105125-A and NHRI-IACUC-105139-A).

Provenance and peer review Not commissioned; externally peer reviewed.

Data availability statement All data relevant to the study are included in the article or uploaded as supplementary information.

Open access This is an open access article distributed in accordance with the Creative Commons Attribution Non Commercial (CC BY-NC 4.0) license, which permits others to distribute, remix, adapt, build upon this work non-commercially, and license their derivative works on different terms, provided the original work is properly cited, appropriate credit is given, any changes made indicated, and the use is non-commercial. See http://creativecommons.org/licenses/by-nc/4.0/.

\section{ORCID iD}

Ming-Hsi Huang http://orcid.org/0000-0002-9670-3821

\section{REFERENCES}

1 Ogra PL, Faden H, Welliver RC. Vaccination strategies for mucosal immune responses. Clin Microbiol Rev 2001;14:430-45.

2 Buchon N, Broderick NA, Lemaitre B. Gut homeostasis in a microbial world: insights from Drosophila melanogaster. Nat Rev Microbiol 2013;11:615-26.

3 Wilson-Welder JH, Torres MP, Kipper MJ, et al. Vaccine adjuvants: current challenges and future approaches. J Pharm Sci 2009;98:1278-316.

4 Lai C-H, Tang N, Jan J-T, et al. Use of recombinant flagellin in oilin-water emulsions enhances hemagglutinin-specific mucosal IgA production and IL-17 secreting T cells against H5N1 avian influenza virus infection. Vaccine 2015;33:4321-9.

5 Huang M-H, Dai S-H, Chong P. Mucosal delivery of a combination adjuvant comprising emulsified fine particles and LD-indolicidin enhances serological immunity to inactivated influenza virus. Microbes Infect 2016;18:706-9.

6 Huang C-H, Huang C-Y, Cheng C-P, et al. Degradable emulsion as vaccine adjuvant reshapes antigen-specific immunity and thereby ameliorates vaccine efficacy. Sci Rep 2016;6:36732.

7 Lin S-I, Huang M-H, Chang Y-W, et al. Chimeric peptide containing both $B$ and T cells epitope of tumor-associated antigen L6 enhances anti-tumor effects in HLA-A2 transgenic mice. Cancer Lett 2016;377:126-33.

8 Huang $\mathrm{C}-\mathrm{H}$, Huang $\mathrm{C}-\mathrm{Y}$, Huang $\mathrm{M}-\mathrm{H}$. Unsaturated squalene content in emulsion vaccine adjuvants plays a crucial role in ROS-mediated antigen uptake and cellular immunity. Mol Pharm 2018;15:420-9.

9 Mumper RJ, Cui Z, Oyewumi MO. Nanotemplate engineering of cell specific nanoparticles. J Dispers Sci Technol 2003;24:569-88.

10 Wendorf J, Singh M, Chesko J, et al. A practical approach to the use of nanoparticles for vaccine delivery. J Pharm Sci 2006;95:2738-50.

11 Xiang SD, Scholzen A, Minigo G, et al. Pathogen recognition and development of particulate vaccines: does size matter? Methods 2006;40:1-9.

12 Wu HY, Nguyen HH, Russell MW. Nasal lymphoid tissue (NALT) as a mucosal immune inductive site. Scand J Immunol 1997;46:506-13.

13 Tadros T, Izquierdo P, Esquena J, et al. Formation and stability of nano-emulsions. Adv Colloid Interface Sci 2004;108-109:303-18.

14 Ohno H, Hase K. Glycoprotein 2 (GP2): grabbing the FimH bacteria into M cells for mucosal immunity. Gut Microbes 2010;1:407-10.

15 Poncet N, Halley PA, Lipina C, et al. Wnt regulates amino acid transporter SLC7A5 and so constrains the integrated stress response in mouse embryos. EMBO Rep 2020;21:e48469.

16 Günzel D, Yu ASL. Claudins and the modulation of tight junction permeability. Physiol Rev 2013;93:525-69.

17 Lee SH, Girard S, Macina D, et al. Susceptibility to mouse cytomegalovirus is associated with deletion of an activating natural killer cell receptor of the C-type lectin superfamily. Nat Genet 2001;28:42-5.

18 Lefranc MP. Nomenclature of the human immunoglobulin kappa (IGK) genes. Exp Clin Immunogenet 2001;18:161-74.

19 Freud AG, Zhao S, Wei S, et al. Expression of the activating receptor, NKp46 (CD335), in human natural killer and T-cell neoplasia. Am J Clin Pathol 2013;140:853-66. 
20 Li AV, Moon JJ, Abraham W, et al. Generation of effector memory T cell-based mucosal and systemic immunity with pulmonary nanoparticle vaccination. Sci Trans/ Med 2013;5:204ra130.

21 Black M, Trent A, Kostenko Y, et al. Self-Assembled peptide amphiphile micelles containing a cytotoxic T-cell epitope promote a protective immune response in vivo. Adv Mater 2012;24:3845-9.

22 Alter G, Malenfant JM, Altfeld M. CD107a as a functional marker for the identification of natural killer cell activity. J Immunol Methods 2004;294:15-22.

23 Ugozzoli M, O'Hagan DT, Ott GS. Intranasal immunization of mice with herpes simplex virus type 2 recombinant GD2: the effect of adjuvants on mucosal and serum antibody responses. Immunology 1998;93:563-71.

24 Kirch J, Guenther M, Doshi N, et al. Mucociliary clearance of microand nanoparticles is independent of size, shape and charge--an ex vivo and in silico approach. $J$ Control Release 2012;159:128-34.

25 Kamaly N, Xiao Z, Valencia PM, et al. Targeted polymeric therapeutic nanoparticles: design, development and clinical translation. Chem Soc Rev 2012;41:2971-3010.

26 Xiao Z, Su Z, Han S, et al. Dual pH-sensitive nanodrug blocks PD-1 immune checkpoint and uses T cells to deliver NF- $\mathrm{KB}$ inhibitor for antitumor immunotherapy. Sci Adv 2020;6:eaay7785.

27 de Pablo MA, Alvarez de Cienfuegos G. Modulatory effects of dietary lipids on immune system functions. Immunol Cell Biol 2000;78:31-9.

28 Clamp AG, Ladha S, Clark DC, et al. The influence of dietary lipids on the composition and membrane fluidity of rat hepatocyte plasma membrane. Lipids 1997;32:179-84.
29 Ahn YK, Kim JH. Effects of squalene on the immune responses in mice(II): Cellular and non-specific immune response and antitumor activity of squalene. Arch Pharm Res 1992;15:20-9.

30 Gaudin A, Song E, King AR, et al. PEGylated squalenoyl-gemcitabine nanoparticles for the treatment of glioblastoma. Biomaterials 2016;105:136-44.

31 Huang C-Y, Huang C-H, Liu S-J, et al. Polysorbasome: a colloidal vesicle contoured by polymeric bioresorbable amphiphiles as an immunogenic depot for vaccine delivery. ACS Appl Mater Interfaces 2018;10:12553-61.

32 Bielinska AU, Janczak KW, Landers JJ, et al. Mucosal immunization with a novel nanoemulsion-based recombinant anthrax protective antigen vaccine protects against Bacillus anthracis spore challenge. Infect Immun 2007;75:4020-9.

33 Hamouda T, Sutcliffe JA, Ciotti S, et al. Intranasal immunization of ferrets with commercial trivalent influenza vaccines formulated in a nanoemulsion-based adjuvant. Clin Vaccine Immunol 2011;18:1167-75.

34 Passmore C, Makidon PE, O'Konek JJ, et al. Intranasal immunization with W 80 5EC adjuvanted recombinant RSV rF-ptn enhances clearance of respiratory syncytial virus in a mouse model. Hum Vaccin Immunother 2014;10:615-22.

35 Guillerey C, Huntington ND, Smyth MJ. Targeting natural killer cells in cancer immunotherapy. Nat Immunol 2016;17:1025-36.

36 Kryczek I, Wei S, Szeliga W, et al. Endogenous IL-17 contributes to reduced tumor growth and metastasis. Blood 2009;114:357-9. 